\title{
Airway narrowing in porcine bronchi with and without lung parenchyma
}

\author{
P.B. Noble, A. Sharma, P.K. McFawn and H.W. Mitchell
}

\begin{abstract}
During bronchoconstriction elastic after-loads arise due to distortion of lung parenchyma by the narrowing airway. In the present study, the functional effect of parenchymal elastic after-load on airway narrowing was determined.
\end{abstract}

Airway narrowing was measured in vivo over a range of transpulmonary pressures and compared with in vitro narrowing measured at corresponding transmural pressures. Bronchi were generation 10 with internal diameters of $\sim 4 \mathrm{~mm}$. In vivo luminal narrowing was measured by videobronchoscopy in anaesthetised and ventilated pigs. In vitro luminal narrowing was measured by videoendoscopy in isolated bronchial segments. Airways were activated by maximum vagal nerve stimulation and maximum electrical field stimulation in vivo and in vitro, respectively.

At $5 \mathrm{cmH}_{2} \mathrm{O}$, stimulation produced a $35.9 \pm 3.2 \%(n=6)$ and a $36.5 \pm 2.4 \%(n=11)$ decrease in lumen diameter in vivo and in vitro, respectively. At $30 \mathrm{cmH}_{2} \mathrm{O}$, luminal narrowing fell to $23.7 \pm 2.0 \%$ in vivo and $23.4 \pm 2.5 \%$ in vitro. There was no difference between luminal narrowing in vivo and in vitro at any pressure.

In conclusion, these findings suggest that in mid-sized, cartilaginous bronchi, parenchymal elastic after-loads do not restrict airway narrowing.

\section{KEYWORDS: Airway narrowing, mechanical loads, parenchymal forces}

$\mathbf{T}$ he degree by which airways narrow in response to bronchoconstrictor stimuli is largely determined by a balance between airway smooth muscle (ASM) contraction and opposing mechanical loads [1]. The after-loads that oppose narrowing consist of transmural pressure $(P \mathrm{tm}$; which is also the pre-load on ASM) and elastic after-loads, which develop as narrowing progressively distorts tissue structures. These elastic after-loads arise both from within the airway wall and from surrounding lung parenchyma [1]. Narrowing of isolated bronchi is reduced by wall stiffness, suggesting that airway wall structures place mechanical loads on ASM limiting narrowing [2]. Outside the airway, elastic after-loads arise due to distortion of lung parenchyma by the narrowing airway [3, 4], often referred to as parenchymal interdependence. Mathematical analyses of airway narrowing suggest that elastic loads produced by parenchymal distortion are sufficient in magnitude to restrict airway narrowing, particularly at low lung volumes [5]. The effects of parenchymal elastic after-loads on airway narrowing can also be inferred from comparisons of

For editorial comments see page 759 . airway narrowing in the lung and in excised bronchial segments. Canine airway segments, from which all surrounding parenchyma is removed, show greater narrowing than similar airways in the lung [6,7], suggesting that the lung limits airway narrowing in vivo. However, a recent study by BROWN and MITZNER [8] demonstrated that in vivo airways can narrow to closure, questioning the capacity of parenchymal forces to restrict airway narrowing.

Mid- and large-sized airways make major contributions to airway resistance in animals $[9,10]$. Furthermore, evoked changes in the calibre of these conducting airways make a substantial contribution to airway responsiveness in healthy humans [11, 12]. It has been suggested that parenchymal load may be reduced in asthma, which may lead to the exaggerated narrowing of airways contributing to the increased airway resistance seen in this disease [13]. However, some morphological properties of larger airways, such as a thick outer wall and stiffness, may limit the magnitude of elastic after-loads during evoked narrowing in airway generations thought to be involved in responsiveness.

The aim of the present study was to assess the significance of parenchymal elastic after-loads

\section{AFFILIATIONS}

Physiology, School of Biomedical, Biomolecular and Chemical Sciences, University of Western Australia, Crawley, Australia.

CORRESPONDENCE

P.B. Noble

Physiology

School of Biomedical and Chemical Sciences

University of Western Australia

35 Stirling Highway

Crawley

Perth

Western Australia 6009

Australia

Fax: 61864881025

E-mail:

noblep01@cyllene.uwa.edu.au

Received:

June 062005

Accepted after revision:

August 092005

SUPPORT STATEMENT

This study was supported by funding from NH\&MRC (Australia).

Online ISSN 1399-3003 
to narrowing of mid-sized bronchi. Airway narrowing was measured in the lungs of anaesthetised pigs and in excised airways matched for location and mode of stimulation. Airway segments were maintained in a simulated in vivo environment by controlling for changes in airway length and circumference produced by breathing movements. To the current authors' knowledge, no studies are reported in which airway narrowing has been recorded in the same airway generation both with and without normal parenchymal attachments, and with a near identical length and volume history. Airway narrowing to maximal nerve stimulation was measured directly using digital bronchoscopy and endoscopy techniques. As the isolated airways had all parenchyma removed, it is argued that any differences between airway narrowing in vivo and in vitro are due to the effects of parenchymal elastic after-loads.

\section{METHODS}

\section{Animal handling}

All animal experiments conducted conformed to the 'Helsinki convention for the use and care of animals'. Experiments were approved by an institutional ethics and animal care unit. Female pigs, $25 \mathrm{~kg}$ in weight, were initially sedated with tiletamine/zolazepam ( $4.4 \mathrm{mg} \cdot \mathrm{Kg}^{-1}$ intramuscularly) and xylazine $\left(2.2 \mathrm{mg} \cdot \mathrm{kg}^{-1}\right.$ intramuscularly). Animals were then used for either in vivo experimentation $(n=6)$ or for in vitro studies $(\mathrm{n}=11)$. After in vivo experimentation, animals were sacrificed using an overdose of pentobarbitone sodium $\left(160 \mathrm{mg} \cdot \mathrm{kg}^{-1}\right)$. Animals used for in vitro studies were exsanguinated under pentobarbitone sodium anaesthesia $\left(30 \mathrm{mg} \cdot \mathrm{kg}^{-1}\right)$.

\section{Surgery and in vivo preparation}

Surgical procedures were carried out under thiopentone sodium $\left(5 \mathrm{mg} \cdot \mathrm{kg}^{-1} \cdot \mathrm{h}^{-1}\right)$ and fentanyl citrate $\left(5 \mu \mathrm{g} \cdot \mathrm{kg}^{-1}\right.$ induction; $20 \mu \mathrm{g} \cdot \mathrm{kg}^{-1} \cdot \mathrm{h}^{-1}$ maintenance) anaesthesia administered via an ear vein drip. The level of anaesthesia was assessed from an ECG and arterial blood pressure, measured though a catheter placed in a femoral artery. Following the induction of anaesthesia, a tracheostomy was performed and pigs were mechanically ventilated (model 708; Harvard Apparatus, Edenbridge, UK) via an endotracheal tube. Arterial blood gases were measured using a blood gas analyser (Rapidlab 800; Bayer, Leverkusen, Germany) and ventilator settings were adjusted to maintain carbon dioxide arterial tension between 4.6-5.3 $\mathrm{kPa}$ (35-40 mmHg). A bilateral pneumothorax was induced and pigs were ventilated with a positive end expiratory pressure of $5 \mathrm{cmH}_{2} \mathrm{O}$.

Lung volume and pressure was measured using a PowerLab recording system (ADInstruments, Castle Hill, Australia). Flow was determined via a pneumotachograph (3500 series; Hans Rudolph, Kansas City, MO, USA) and a differential pressure transducer (MPX2010DP; Motorola Semiconductors, Phoenix, AZ, USA) connected to the end of the endotracheal tube. Tidal volume was integrated from the flow signal. Transpulmonary pressure $(P$ tp $)$ was recorded from the difference between oesophageal and airway opening pressures. Oesophageal and airway opening pressures were recorded by connecting one side of a differential pressure transducer to an air filled oesophageal cannula, positioned with the distal end in the mid-thoracic oesophagus and the other to a line fed from the endotracheal tube (airway opening pressure). In the presence of a pneumothorax, $P_{t p}$ and airway opening pressures were the same.

\section{Bronchoscopy}

Airway diameter (resting and after maximal nerve stimulation, see below) was recorded using an Olympus bronchoscope (IT10; Olympus, Melville, NY, USA) and a digital camera (Firei400; Unibrain, San Ramon, CA, USA). To allow for ventilation during bronchoscopic evaluation, a swivel-Y connector (Bodai PEEP-safe; Sontec Medical Inc., Denver, CO, USA) was inserted between the endotracheal tube and the pneumotachograph. One end of the $\mathrm{Y}$ connector was sealed with a polypropylene and silicone rubber membrane providing an airtight fit around the bronchoscope. The bronchoscope was inserted into the left lung to generation 10 of the stem bronchus, determined by counting side branches as the bronchoscope was inserted (trachea $=0$ ). The airway lumen was marked using steel ball bearings $(0.5 \mathrm{~mm}$ in diameter $)$ inserted through a bronchoscope channel.

The left vagus nerve was exposed in the neck and ligated. A skin pouch was formed and filled with paraffin oil to prevent the nerve from drying out. Airway narrowing was induced by vagal stimulation (Grass S44 square-wave stimulator; Grass Intruments Co., Quincy, MA, USA) via platinum electrodes placed directly under the nerve caudal to the ligature. Stimulation parameters used $(20 \mathrm{~V}, 0.5 \mathrm{~ms}$ and $30 \mathrm{~Hz})$ produced maximal activation of the vagus nerve. No further increase in airway narrowing was observed when stimulus parameters were increased in preliminary experiments. Vagal stimulation resulted in cardiac arrest assessed from femoral blood pressure recordings (fig. 1). A test stimulation was initially conducted and if cardiac arrest was not observed electrodes were re-positioned. Animals not showing complete cardiac arrest on vagal stimulation were excluded. Pigs were given propranalol $\left(0.5 \mathrm{mg} \cdot \mathrm{kg} \cdot \mathrm{h}^{-1}\right)$ to minimise possible effects of catecholamine release on airway responses [14].

Vagal stimulations were carried out with lungs inflated to a range of $P$ tps $\left(5,10,15,20\right.$ and $30 \mathrm{cmH}_{2} \mathrm{O}$, in a randomised order). Lungs were inflated by connecting the endotracheal

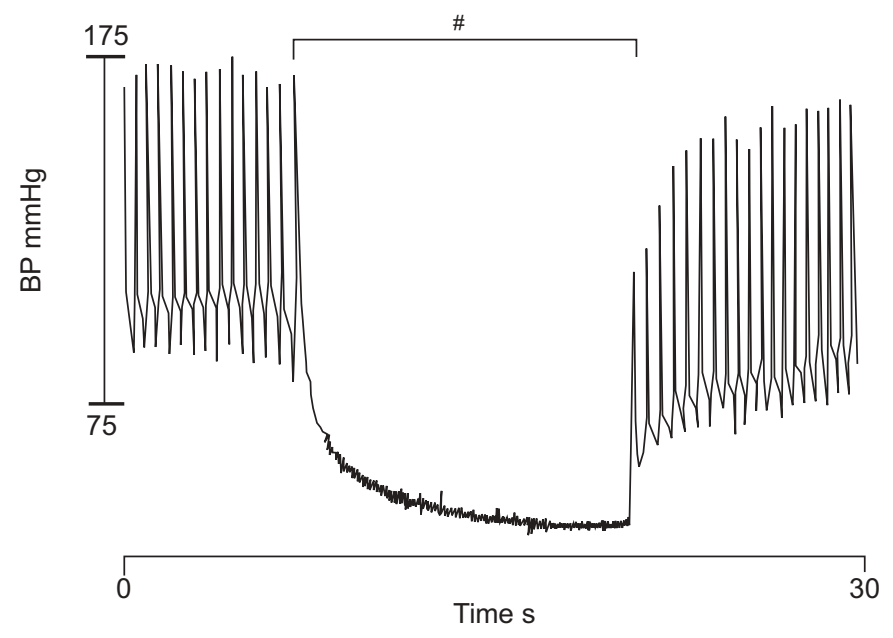

FIGURE 1. Diagram showing vagal stimulation (\#; $20 \mathrm{~V}, 0.5 \mathrm{~ms}, 30 \mathrm{~Hz}$ ) on femoral arterial blood pressure (BP). 
tube to a 40-L plastic chamber, which was pressurised with compressed air. Lungs were twice inflated to $30 \mathrm{cmH}_{2} \mathrm{O} P$ tp to standardise volume history, $2 \mathrm{~min}$ before stimulation. Immediately preceding vagal stimulation, the ventilator was switched off and lungs were inflated to the desired pressure. Vagal stimulation was maintained until no further airway narrowing was observed, which was typically 15-20 s.

The effect of $P$ tp on bronchial length was measured so that corresponding length changes could be duplicated in vitro. Bronchial and tracheal lengths were measured at each $P_{\text {tp }}$ used. Total airway length (bronchial plus tracheal length) was calculated from the length of bronchoscope inserted into the lung (left or right) plus the distance between the ball-bearing marker and the bronchoscope lens. The distance to the marker was determined from the size of the marker in the image compared with calibrated images recorded at known distances. Tracheal length was measured from the end of the endotracheal tube to an additional marker placed at the bifurcation. At each $P$ tp tracheal length was subtracted from total airway length to give bronchial length (from the bifurcation to the marker). Airway lengths were measured after stepwise deflations from 30 to $0 \mathrm{cmH}_{2} \mathrm{O} P$ tp.

\section{Bronchial segment preparation}

In a separate group of animals, bronchial segments from the left lung were dissected free of parenchyma and all had side branches ligated. In order to keep the same bronchial length in vitro as occurred in vivo, bronchial length was measured from the bifurcation to the 10th generation (where luminal narrowing was recorded). The length of isolated bronchi at $0 \mathrm{cmH}_{2} \mathrm{O}$ $P \mathrm{tm}$ was the same as that measured in the lung in vivo by bronchoscopy at $0 \mathrm{cmH}_{2} \mathrm{O} P$ tp $(65.4 \pm 0.7 \mathrm{~mm}$ in vitro versus $61.8 \pm 2.4 \mathrm{~mm}$ in vivo), suggesting that there was no recoil in the length of bronchus after dissection. Once the distance from bifurcation to the 10th generation was measured, a segment of bronchus extending $2.5 \mathrm{~cm}$ to either side of the 10th generation was taken (total length $5 \mathrm{~cm}$ ). Bronchial segments were cannulated at each end and placed horizontally in an organ bath containing gassed $\left(95 \% \mathrm{O}_{2}, 5 \% \mathrm{CO}_{2}\right)$ Krebs solution (mM: $\mathrm{NaCl} 121 ; \mathrm{KCl} 5.4 ; \mathrm{MgSO}_{4} 1.2 ; \mathrm{NaHCO}_{3} 25$; sodium morpholinopropane sulphonic acid 5.0; glucose 11.5; and $\mathrm{CaCl}_{2} 2.5$ ) at $37^{\circ} \mathrm{C}$. The length of the segment was initially set to $5 \mathrm{~cm}$, i.e. the same length as in vivo at $0 \mathrm{cmH}_{2} \mathrm{O} P$ tp. The airway lumen was flushed via a jacketed reservoir containing Krebs solution. The height of Krebs solution within the reservoir set the $P \mathrm{tm}$ of the segment. Krebs solution contained propranalol $\left(10^{-6} \mathrm{M}\right)$.

\section{Endoscopy}

Airway diameter was recorded using a rigid fibreoptic endoscope (1711D; Olympus Selfscope SES) and a digital camera (DFW-SX900; Sony, Park Ridge, NJ, USA). Methods for recording airway narrowing using an endoscope are described in detail elsewhere [15]. Briefly, the region of interest was stained with a blue dye using a steel applicator. The region selected was the same as that used in vivo. The endoscope was inserted into the lumen and sealed by a latex membrane. Airway narrowing was induced by electric field stimulation (EFS) using platinum electrodes. Stimulation parameters were chosen to produce maximum airway narrowing to nerve stimulation $(300 \mathrm{~mA}, 3 \mathrm{~ms}$ and $30 \mathrm{~Hz})$, that is increasing stimulus parameters did not produce any further narrowing. Images were calibrated with a $1-\mathrm{mm}$ diameter probe inserted into the distal end of the segment.

Following preparation, bronchial segments were equilibrated for $1 \mathrm{~h}$ and the viability of the bronchus confirmed to $10^{-4} \mathrm{M}$ acetylcholine (ACh). Following recovery, EFS was applied every $10 \mathrm{~min}$ with airways inflated to 5, 10, 15, 20 and $30 \mathrm{cmH}_{2} \mathrm{O} \mathrm{Ptm}_{\mathrm{tm}}$ in randomised order. Stimulation was maintained until no further increase in narrowing was observed ( $\sim 20 \mathrm{~s})$. Airway segments were stimulated at a similar length as airways in vivo, and were exposed to a similar volume history. At each $P$ tm bronchial segments were stretched by the same percentage observed at the corresponding $P_{\mathrm{tp}}$ in vivo (see Results section). Between stimulations, $P$ tm was oscillated at amplitudes and frequencies similar to that observed with ventilation in vivo $\left(5-18 \mathrm{cmH}_{2} \mathrm{O}\right.$ at $\left.0.5 \mathrm{~Hz}\right) . P$ tm oscillation was carried out by cycling the height of Krebs solution in the reservoir using an elliptical piston pump. Airways were twice inflated to $30 \mathrm{cmH}_{2} \mathrm{O} \mathrm{Ptm}, 2 \mathrm{~min}$ before stimulation.

\section{Compliance}

In a separate group of animals, pressure-volume curves were constructed at room temperature in both excised bronchial segments (from left lungs) and intact lower lung lobes (left) from 0 to $30 \mathrm{cmH}_{2} \mathrm{O}$. Pressures in the airway or lung were monitored with a pressure transducer. Bronchial segments filled with Krebs solution were inflated using a microsyringe driven with a stepping motor controlled by computer software. The initial volume of segments at atmospheric pressure was measured from the volume of solution, required to fill the segment [16]. Lung lobes were cannulated and inflated with air using a $60-\mathrm{mL}$ syringe. The surface of the lung was kept moist with Krebs solution. The initial volume of gas in the lung was calculated from the lobe volume, measured by water displacement, minus the lobe mass ( $\mathrm{g}$ ) and assuming a tissue specific gravity of $1.06[17,18]$. At each pressure, gas volumes were corrected for compression using Boyle's law. Pressure was recorded after $>90 \%$ of the stress relaxation had occurred. Airway segments and lung lobes were cycled until a consistent pressure-volume curve was obtained and this was used for analysis. The changes in volume at each pressure were normalised for the volume at $0 \mathrm{cmH}_{2} \mathrm{O}$ (i.e. strain $=\Delta V / \Delta V 0$ ). Specific compliance $\left(\mathrm{cmH}_{2} \mathrm{O}^{-1}\right)$ was determined from the deflationary limb of the linear portion of the strain-pressure curve. The linear region of the curve was determined by calculating the correlation coefficient and data points were removed from the ends of the curve until $r^{2}$ was $>0.99$.

\section{Analysis and statistics}

Luminal cross-sectional areas in resting and contracted airways were quantified by tracing an area around the lumen of the airway using Optimas image analysis software (Media Cybernetics, Silver Spring, MD, USA). Calibration markers were also measured (ball bearings in vivo or probe in vitro), which allowed conversion of area measurements from pixels ${ }^{2}$ to $\mathrm{mm}^{2}$. Airway diameters were determined from crosssectional areas assuming a circular lumen [15]. For images recorded through the bronchoscope, measurements required correction for radial image distortion [19]. To correct for distortion, a recently developed method was used in which 
a grid with 1-mm squares is viewed underneath the bronchoscope and correction functions are generated by curve fitting between known and distorted dimensions [20]. To determine the effectiveness of the generated equations in correcting for lens distortion, discs of known diameters were viewed underneath the bronchoscope. Before mathematical correction, the diameters of discs viewed at the periphery of the lens were distorted by $\sim 20 \%$, compared with discs viewed at the centre of the lens (where distortion is minimal). After mathematical correction, the error in diameter measurements was reduced to $<1 \%$.

Comparisons between lumen diameters, luminal narrowing responses and airway lengths at different pressures were made using paired or unpaired ANOVA, as appropriate. Mean compliances of airways and lobes were compared using unpaired t-test. Data are given as mean $\pm S E$, where $n$ equals the number of animals. A p-value of $<0.05$ was considered statistically significant.

\section{RESULTS}

Resting lumen diameters, before nerve stimulation, were $3.7 \pm 0.2 \mathrm{~mm}$ in vivo $(\mathrm{n}=6)$ and $3.8 \pm 0.2 \mathrm{~mm}$ in vitro $(\mathrm{n}=11)$ at $5 \mathrm{cmH}_{2} \mathrm{O}$. Maximum inflation to $30 \mathrm{cmH}_{2} \mathrm{O}$ increased resting lumen diameters to $4.9 \pm 0.2 \mathrm{~mm}$ in vivo $(\mathrm{p}<0.001)$ and $4.6 \pm 0.2 \mathrm{~mm}$ in vitro $(\mathrm{p}<0.001)$. There was no significant difference between resting lumen diameters recorded in vivo and in vitro at any pressure $(\mathrm{p}=0.6$; fig. 2$)$.

Example images of airway lumens recorded in vivo and in vitro, before and after nerve stimulation, are shown in figure 3. Maximal nerve stimulation at $5 \mathrm{cmH}_{2} \mathrm{O}$ produced a $35.9 \pm 3.2 \%$ and $36.5 \pm 2.4 \%$ decrease in lumen diameter in vivo and in vitro, respectively. Luminal narrowing was not significantly different between pressures of 5 and $15 \mathrm{cmH}_{2} \mathrm{O}$ in either in vivo or in vitro airways, but was reduced at higher pressures (fig. 4). At $30 \mathrm{cmH}_{2} \mathrm{O}$, luminal narrowing fell to $23.7 \pm 2.0 \%$ in vivo

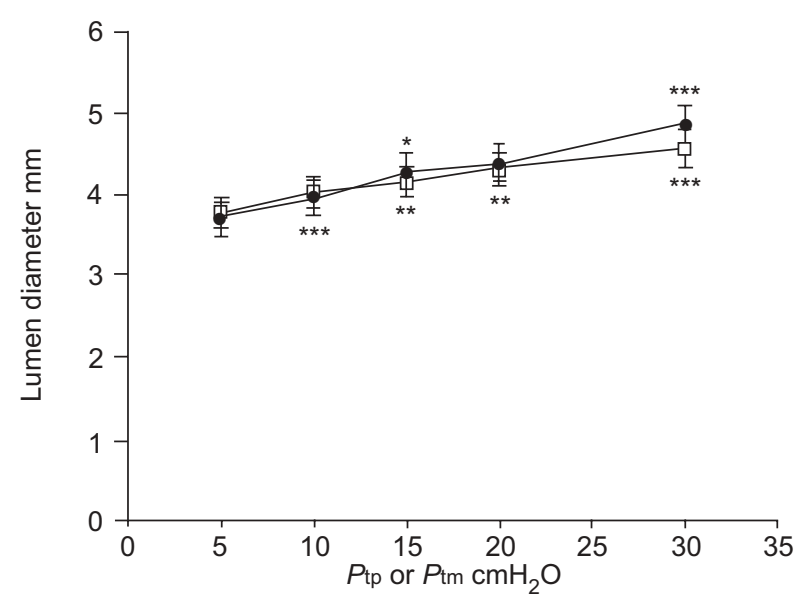

FIGURE 2. Mean lumen diameter versus pressure recorded in lungs in vivo ( $n=6)$ and airway segments in vitro $(\square ; n=11)$. There was a significant effect of inflation pressure on lumen diameter in airways both in vivo and in vitro. However, there was no significant difference between lumen diameter measured in vivo and in vitro at any pressure $(\mathrm{p}=0.6)$. Ptp: transpulmonary pressure; $P$ tm: transmural pressure. ${ }^{*}: p<0.05 ; * *: p<0.01 ; * * *: p<0.001$ compared with preceding lower pressure.

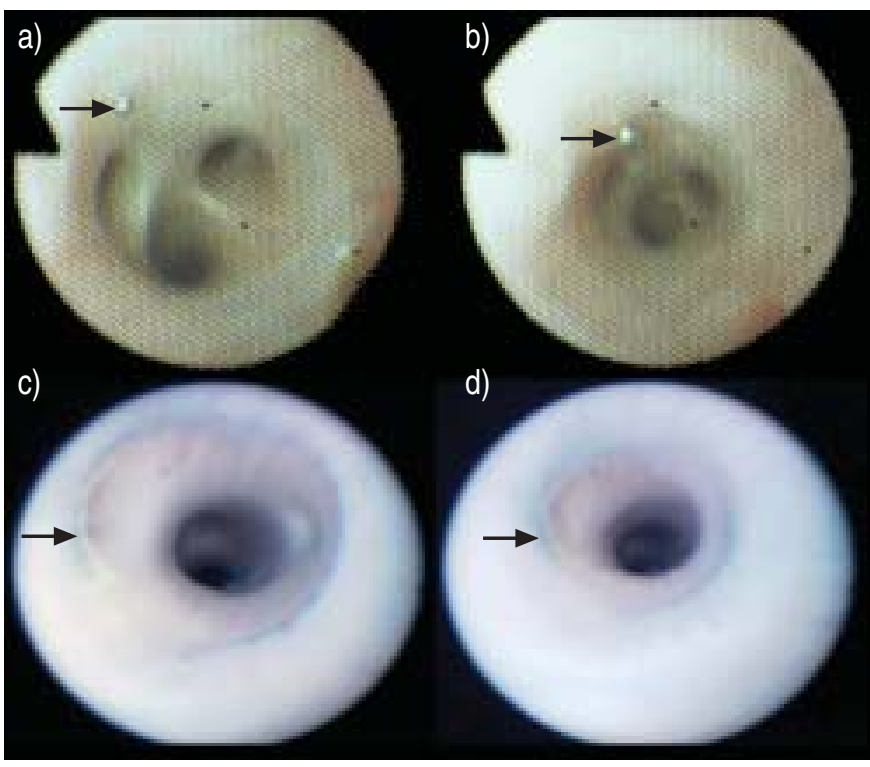

FIGURE 3. Example images of the airway lumen recorded in vivo $(a, b)$ and in vitro ( $c, d)$ before $(a, c)$ and after $(b, d)$ maximal nerve stimulation. The airway lumen was visualised by bronchoscopy in vivo and endoscopy in vitro. Arrows indicate spherical markers in vivo that were used for calibration purposes, and the dye that was applied in vitro to aid recording

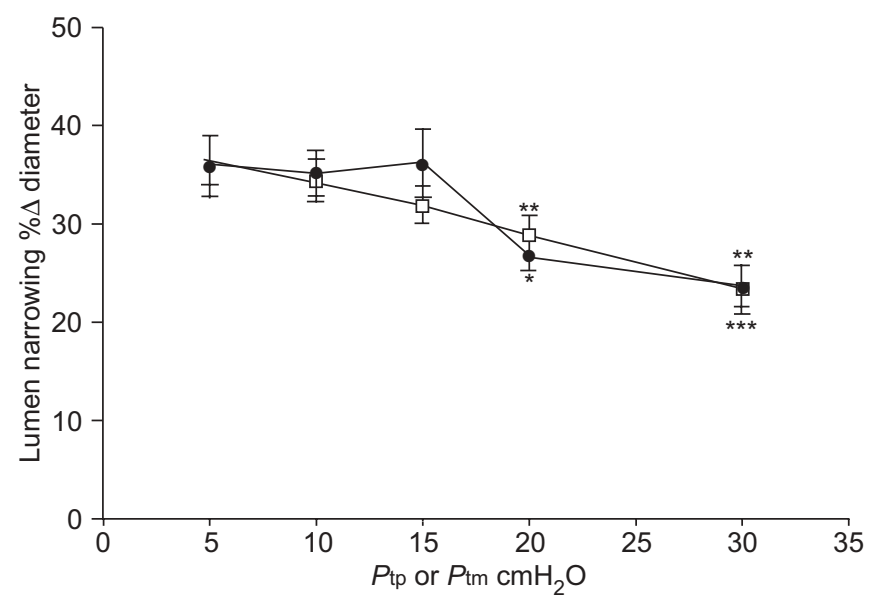

FIGURE 4. Airway narrowing recorded in lungs in vivo $(\bullet ; n=6)$ and airway segments in vitro $(\square ; n=11)$ following maximal nerve stimulation. Airway narrowing was determined over a range of pressures (transpulmonary pressure $(P \mathrm{tp})$ or transmural pressure $(P \mathrm{tm}))$. Narrowing was quantified by the percentage change $(\% \triangle)$ in lumen diameter. Luminal narrowing was reduced at higher pressures in both in vivo and in vitro airways. However, there was no significant difference between luminal narrowing measured in vivo and in vitro at any pressure $(p=0.7)$. ${ }^{*}: \mathrm{p}<0.05 ;{ }^{* *}: \mathrm{p}<0.01 ; * * *: \mathrm{p}<0.001$ compared with $5 \mathrm{cmH}_{2} \mathrm{O}$.

$(\mathrm{p}<0.01)$ and $23.4 \pm 2.5 \%$ in vitro $(\mathrm{p}<0.001)$. There was no significant difference in luminal narrowing recorded in vivo and in vitro at any pressure $(\mathrm{p}=0.7)$.

Typical pressure-strain curves for a lower lung lobe and an airway segment are shown in figure 5. Lung lobes had greater strain and exhibited greater hysteresis than airway segments. Lung compliance was $>3$-fold greater than airway compliance 


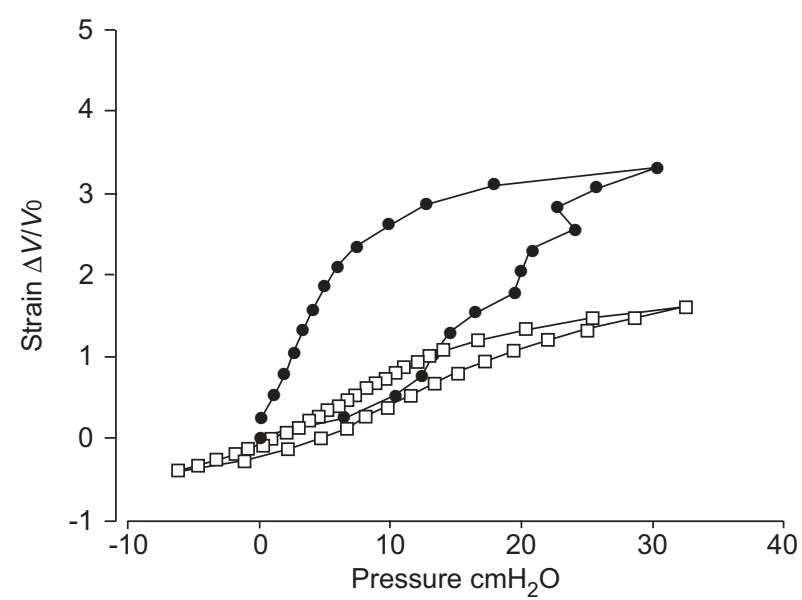

FIGURE 5. A typical strain-pressure curve for a lower lung lobe $(\bullet)$ and a bronchus segment $(\square)$. Inflationary (lower) and deflationary (upper) curves are shown for each. $\Delta V$ : change in volume; $V 0$ : volume at $0 \mathrm{cmH}_{2} \mathrm{O}$.

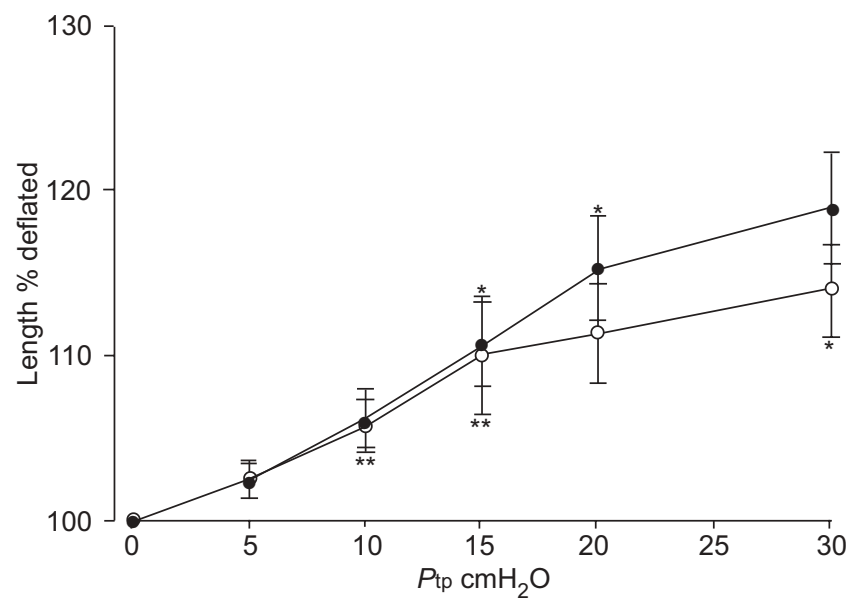

FIGURE 6. Relationship between airway length and transpulmonary pressure $(P$ tp) for bronchi $(\bullet ; n=8)$ and tracheas $(O ; n=8)$. Airway lengths were expressed as a percentage of their deflated length at $0 \mathrm{cmH}_{2} \mathrm{O}$. Airway inflation produced significant lengthening in both bronchi and tracheas. Percentage length changes were not significantly different between bronchi and tracheas $(p=0.2)$. *: $p<0.05$; $* *$ : $p<0.01$ compared with preceding lower pressure.

$(\mathrm{p}<0.01)$. Compliance, as determined from the deflationary limb of the strain-pressure curve, was $0.239 \pm 0.029 \mathrm{~cm} \mathrm{H}_{2} \mathrm{O}^{-1}$ and $0.069 \pm 0.006 \mathrm{cmH}_{2} \mathrm{O}^{-1}$ in lungs $(\mathrm{n}=4)$ and airways $(\mathrm{n}=4)$, respectively.

Lung inflation produced significant airway lengthening. Inflation from 0 to $30 \mathrm{cmH}_{2} \mathrm{O}$ stretched bronchi by $18.9 \pm 3.4 \%$ $(\mathrm{p}<0.001)$ and tracheas by $14.0 \pm 2.8 \%(\mathrm{p}<0.001)$. The percentage change in airway length with lung inflation was not significantly different between bronchi and tracheas $(p=0.2$; fig. $6 ; n=8)$.

\section{DISCUSSION}

Airways in vivo are exposed to after-loads arising from the airway wall and lung parenchyma. These include transmural loads produced by lung inflation (i.e. $P$ tm), and elastic loads from the airway wall and lung parenchyma, which arise from distortion of tissue structures by the narrowing airway. The primary aim of the present study was to determine the functional effect of elastic after-loads produced by distortion of the lung parenchyma during bronchoconstriction. In excised bronchial segments, airway wall and transmural loads were preserved; however, parenchymal elastic after-loads were eliminated because airways were separated from all parenchymal attachments. This experimental approach allowed the narrowing capacity of airways to be assessed in the presence and absence of parenchymal elastic after-loads. Using a novel method, airways in vitro were maintained in an environment simulating in vivo conditions by controlling for changes in airway length and cyclical lumen dilation, produced by breathing movements. The present results showed that there was no difference in the degree of airway narrowing measured in the lung or in excised airways at any lung volume, suggesting that elastic after-loads from the parenchyma did not provide a significant mechanical load on airway narrowing of mid-sized bronchi.

In the healthy state, mid-sized bronchi make a major contribution to airway responsiveness [9-12]. Given a weak or absent effect of parenchyma on narrowing in these airway generations, the current authors suggest that elastic after-loads do not make a major contribution to airway responsiveness in the normal lung. Mid-sized bronchi are also a major site of airway remodelling in severe asthma [21], implicating them in the exaggerated airway narrowing associated with lung disease (i.e. airway hyperresponsiveness (AHR)). If these airways do contribute to AHR, then the present findings further suggest that hyperresponsiveness may not be caused by uncoupling of the airway wall from parenchymal elastic after-loads, as previously suggested [13]. Smaller peripheral airways are also involved in asthma pathophysiology with major consequences to gas exchange in the event of airway closure. Further, studies suggest that peripheral airway narrowing, by way of a heterogenous airway constriction, can induce AHR [22]. The effect of parenchymal elastic afterloads on airway narrowing in small peripheral airways is not clear; however, their importance has also been questioned [22].

The absence of an effect of parenchymal elastic after-load on airway narrowing in the present study is most likely due to reduced outer airway wall narrowing, which effectively uncouples ASM from parenchymal load. Several properties of the airway wall may restrict outer airway wall movement. As a result of the wall geometry, thick-walled airways have less adventitial narrowing than thinner-walled airways for the same degree of luminal narrowing. Consequently, the magnitude of parenchymal elastic after-load developed on bronchi may be small compared with bronchioles. Another property of the airway wall that may reduce the parenchymal elastic afterload is airway wall stiffness. The importance of parenchymal elastic after-loads may be smaller for airways with low wall compliance due to restricted movement of the outer airway wall. The present study showed that bronchial segments were three times as stiff as lung tissue. Despite having a stiff airway wall with minimal narrowing of the adventitia, the bronchial lumen can still narrow by approximately half. The mechanism accounting for this apparently paradoxical situation could be uncoupling of the inner airway wall from the outer wall during 
ASM contraction [23-25]. Some analyses assume that the airway wall cross-sectional area is constant during bronchoconstriction [26]. However, some studies, in excised airways and airways in situ, indicate that the wall area expands due to uncoupling of the inner airway wall from the cartilaginous outer airway wall [24, 25]. Accordingly, there is a greater difference between luminal and adventitial narrowing than expected from wall thickness alone [27]. If airway uncoupling occurred in vivo, as suggested in some studies [23], then substantial lumen narrowing could occur with only minor changes in external airway diameter and little distortion of lung parenchyma.

Previous studies suggest that parenchymal loads provide a significant brake on airway narrowing, although the lung volumes at which parenchymal loads take effect differ between studies. DiNG et al. [28] demonstrated that small changes in lung volume, comparable to tidal volume, produce significant attenuation of methacholine-induced bronchoconstriction in humans. GUNST et al. [7] determined a critical $P$ tp of $7.5 \mathrm{cmH}_{2} \mathrm{O}$, above which parenchymal loads were sufficient to prevent airway closure, and below which airway closure was readily observed. However, the contribution of parenchymal interdependence, i.e. elastic after-loads produced by distortion of parenchyma, and the loads applied by lung inflation to these findings are not known. In the studies by DING et al. [28] and GUNST et al. [7], airway narrowing may be reduced at high lung volumes by an increase in transmural load rather than parenchymal elastic after-load.

The current authors' findings on parenchymal load are consistent with those reported by BROWN and MITZNER [8], which suggest that maximum airway narrowing is more dependent on the mode of drug delivery than on the capacity of parenchymal loads to restrict airway narrowing. When bronchoconstriction is induced by instillation, rather than by the more commonly used aerosol, airway closure is produced at high peak expiratory pressures [29], suggesting that parenchymal loads (including parenchymal elastic after-loads) are insufficient to prevent airway closure even at high lung volumes. However, the present study differed from that of BROWN and MitZNER [8] in dogs as airway closure was not observed in response to maximal stimulation. These disparate findings are most likely due to intrinsic differences between the airways of pigs and dogs rather than as a result of the different methods of provocation (methacholine instillation versus vagal stimulation). Even under conditions of supramaximal ACh instillation, pig bronchi narrow only $\sim 40 \%$ at $5 \mathrm{cmH}_{2} \mathrm{O} P \mathrm{tm}$ [30], which is similar to the narrowing recorded in the present study using neural stimulation. It is not known whether similar-sized human airways narrow to closure under maximal stimulation conditions [31]. The extent of narrowing recorded in dog and pig bronchi may be influenced by airway wall compliance. The compliance of pig bronchi reported in the present and previous studies [2] is less than that observed for dog bronchi [32], and may prevent airway closure due to greater airway wall load [2].

In the current study, airway narrowing was recorded by digital endoscopy (in vitro) and bronchoscopy (in vivo). Quantitative assessment of airway calibre using bronchoscopy was previously limited due to radial image distortion owing to a wide-angled fish-eye lens [19]. To correct for radial image distortion, grid paper was viewed through the bronchoscope and correction functions were generated by curve fitting between the known grid pattern and the distorted pattern, as previously described [20]. The error in diameter measurements was negligible when the generated correction equations were used.

The functional effect of parenchymal elastic after-load was determined by comparing airway narrowing in vivo and in vitro. Therefore, it was essential to control several key variables in the two experimental procedures. First, lumen narrowing was measured in airways from the same generation determined by counting off side branches. The similar diameters of airways in vivo and in vitro confirm a common anatomical location. Secondly, unlike previous studies [2, 33], excised airway segments were exposed to a similar volume history seen in vivo by oscillating $P \mathrm{tm}$ at amplitudes and frequencies mimicking ventilation. The authors recently demonstrated that bronchoconstrictor responses to EFS in porcine bronchial segments are enhanced after periods of pressure oscillation [34], emphasising the importance for recording airway narrowing under similar conditions in vivo and in vitro. The authors also controlled for changes in airway length that occur with lung inflation or deflation by stretching airway segments to lengths determined at each $P_{\text {tp }}$ in vivo. This is a novel approach and necessary because changes in airway length have been shown to regulate bronchoconstriction [35]. Airway lengthening potentially alters the length of ASM in situ, which may alter the contractile response of the airway. To measure the in vivo length of airways at various lung volumes, with the bronchoscope fixed in position, changes in airway length were determined from the size of the calibration marker as it moved away or towards the bronchoscope lens with lung inflation or deflation, respectively. Maximal lung inflation $\left(0\right.$ to $30 \mathrm{cmH}_{2} \mathrm{O}$ $P$ tp) stretched the length of bronchi by $\sim 19 \%$. This degree of airway lengthening is less than that reported by HYATT et al. [36] who found $30 \%$ lengthening in pigs, and by HuGHES et al. [37] who showed $40 \%$ airway lengthening in dogs.

Whilst these experiments were designed to eliminate parenchymal elastic after-loads in vitro, all other loads likely to restrict ASM contraction were similar in vivo and in vitro (i.e. preloads). Under static conditions in vivo, pre-load is applied to the ASM by parenchymal recoil, which is equal to $P$ tp and is transferred to the airway wall as an elastic recoil pressure [38]. Therefore, in a relaxed airway in vivo the effective $P$ tm and preload is equal to $P$ tp. This pre-load was replicated in vitro by applying a hydrostatic $P$ tm to the airway that was matched to the $P$ tp used in vivo. The near identical expansion of the airway lumen in vitro and in vivo suggests that $P_{\mathrm{tm}}$ and $P_{\mathrm{tp}}$ were equivalent, indicating that airways were exposed to the same pre-loads before the introduction of airway narrowing.

Increasing the $P$ tm on airways significantly reduced narrowing both in vivo and in vitro, possibly due to greater after-load imposed by higher Ptms. Whether the Ptm was applied as a hydrostatic pressure in vitro or by parenchymal recoil in vivo made no difference to airway narrowing. Airway lumen diameters increased with applied $P$ tm suggesting that the operating length of the ASM increased. Previous studies have shown that force production in porcine bronchial segments 
falls at $P_{\text {tms }}>10 \mathrm{cmH}_{2} \mathrm{O}$ [39], suggesting that increased preload could also contribute to reduced narrowing.

One assumption of the present study was that the level of ASM activation was similar in both preparations. Since ASM activation by agonists is dependent on the route of drug delivery [8], airway narrowing was induced by direct vagal stimulation in vivo and by EFS in vitro. There is no significant nonadrenergic or noncholinergic innervation to pig bronchi and propranolol was used both in vivo and in vitro to inhibit effects produced by stimulation of sympathetic nerves [14]. Hence, EFS in vitro stimulated the same post-ganglionic parasympathetic neurons that are under vagal control in vivo. The maximum electrical stimulation parameters were used, that is increasing stimulus parameters produced no further lumen narrowing in vivo and in vitro in pilot experiments (data not shown), indicating that the nerves were maximally activated both in vivo and in vitro. If the efficiency of synaptic transmission at the parasympathetic ganglia is low then the stimulus delivered in vitro might have been higher than in vivo. However, this would produce less narrowing in vivo than in vitro and would overestimate the importance of parenchymal elastic after-loads. It cannot explain the apparent absence of any effect of parenchymal elastic after-loads on airway narrowing.

To investigate the level of ASM activation produced by the different modes of nerve stimulation in vivo and in vitro, the active pressure generated by ASM was estimated using a theoretical analysis developed previously by MACKLEM [40]. Briefly, the active stress produced by ASM can be calculated from the magnitude of airway narrowing, the elastic properties of the airway wall, and the increase in peribronchial stress produced by parenchymal deformation (zero in vitro). Macklem's analysis assumes the elastic properties of the airway are the same for changes in $P$ tm and for ASM contraction. Hence, the compliance curves of the airway can be used to estimate the change in pressure between the relaxed and contracted diameters. For airways in vivo the peribronchial stress produced by parenchymal deformation must also be calculated. Peribronchial stress is determined from the fractional decrease in external diameter and the shear modulus of the lung [41], which in relaxed pig lungs is $0.6 P_{\text {tp }}$ [42]. The change in external airway diameter used to calculate peribronchial stress was unpublished data from a previous study where external airway narrowing in response to maximal EFS was directly measured by video imaging [15]. In that study, a $10 \%$ decrease in external airway diameter at $20 \mathrm{cmH}_{2} \mathrm{O}$ was accompanied by a $29 \%$ decrease in lumen diameter, similar to that reported in the present study $(\sim 27 \%$ in vivo and $\sim 29 \%$ in vitro). The active pressure generated by ASM in airways inflated to $20 \mathrm{cmH}_{2} \mathrm{O}$ was computed since the relaxed and contracted diameters were within the range of the data collected from pressure-volume analysis, and accompanying data on adventitial narrowing at this $P$ tm was available [15]. Active pressure generated by ASM in airways inflated to $20 \mathrm{cmH}_{2} \mathrm{O}$ was calculated to be $\sim 22 \mathrm{cmH}_{2} \mathrm{O}$ in vivo and $20 \mathrm{cmH}_{2} \mathrm{O}$ in vitro. The results of this analysis confirm that direct vagal stimulation in vivo and EFS in vitro produced a similar force of ASM contraction.

In summary, parenchymal elastic after-loads produced by distortion of lung parenchyma during bronchoconstriction do not restrict airway narrowing in mid-sized bronchi. Results show that the major source of after-load on airway smooth muscle in mid-sized airways arises from transmural pressure or from deformation of the airway wall, with little or no effect from parenchymal elastic after-loads. These findings suggest that the mechanism producing airway hyperresponsiveness is not associated with uncoupling of parenchymal elastic afterloads in mid- to large-sized airways.

\section{ACKNOWLEDGEMENTS}

The authors would like to thank C.T. Phan for technical assistance during in vivo experiments. The authors would also like to thank P.D. Sly and Z. Hantos for critical discussion on parenchymal force.

\section{REFERENCES}

1 Moreno RH, Hogg JC, Paré PD. Mechanics of airway narrowing. Am Rev Respir Dis 1986; 133: 1171-1180.

2 Noble PB, Turner DJ, Mitchell HW. Relationship of airway narrowing, compliance, and cartilage in isolated bronchial segments. J Appl Physiol 2002; 92: 1119-1124.

3 Hughes JMB, Jones HA, Wilson AG, Grant BJB, Pride NB. Stability of intrapulmonary bronchial dimensions during expiratory flow in excised lungs. J Appl Physiol 1974; 37: 684-694.

4 Sasaki H, Hoppin FG Jr, Takishima T. Peribronchial pressure in excised dog lungs. J Appl Physiol 1978; 45: 858-869.

5 Lambert RK, Paré PD. Lung parenchymal shear modulus, airway wall remodeling, and bronchial hyperresponsiveness. J Appl Physiol 1997; 83: 140-147.

6 Gunst SJ, Stropp JQ, Service J. Mechanical modulation of pressure-volume characteristics of contracted canine airways in vitro. J Appl Physiol 1990; 68: 2223-2229.

7 Gunst SJ, Warner DO, Wilson TA, Hyatt RE. Parenchymal interdependence and airway response to methacholine in excised dog lobes. J Appl Physiol 1988; 65: 2490-2497.

8 Brown RH, Mitzner W. The myth of maximal airway responsiveness in vivo. J Appl Physiol 1998; 85: 2012-2017.

9 Brown R, Woolcock AJ, Vincent NJ, Macklem PT. Physiological effects of experimental airway obstruction with beads. J Appl Physiol 1969; 27: 328-335.

10 Shioya T, Solway J, Munoz NM, Mack M, Leff AR. Distribution of airway contractile responses within the major diameter bronchi during exogenous bronchoconstriction. Am Rev Respir Dis 1987; 135: 1105-1111.

11 Brown RH, Croisille P, Mudge B, Diemer FB, Permutt S, Togias A. Airway narrowing in healthy humans inhaling methacholine without deep inspirations demonstrated by HRCT. Am J Respir Crit Care Med 2000; 161: 1256-1263.

12 Sekizawa K, Yanai M, Shimizu Y, Sasaki H, Takishima T. Serial distribution of bronchoconstriction in normal subjects. Methacholine versus histamine. Am Rev Respir Dis 1988; 137: 1312-1316.

13 Macklem PT. Factors determining bronchial smooth muscle shortening. Am Rev Respir Dis 1991; 143: S47-S48.

14 Connellan DR, Mitchell HW. Transition of functional innervation in the developing porcine airway from nitrergic to catecholaminergic. Br J Pharmacol 1998; 123: 712-718. 
15 Mitchell HW, Sparrow MP. Video-imaging of lumen narrowing; muscle shortening and flow responsiveness in isolated bronchial segments of the pig. Eur Respir J 1994; 7: 1317-1325.

16 McFawn PK, Mitchell HW. Bronchial compliance and wall structure during development of the immature human and pig lung. Eur Respir J 1997; 10: 27-34.

17 Mansell AL, McAteer AL, Oldmixon EH. Mechanical dissociation of bronchi from parenchyma in the immature piglet lung. J Appl Physiol 2000; 89: 228-234.

18 Stengel PW, Frazer DG, Weber KC. Lung degassing: an evaluation of two methods. J Appl Physiol 1980; 48: 370-375.

19 Forkert L, Watanabe H, Sutherland K, Vincent S, Fisher JT. Quantitative videobronchoscopy: a new technique to assess airway caliber. Am J Respir Crit Care Med 1996; 154: 1794-1803.

20 McFawn PK, Forkert L, Fisher JT. A new method to perform quantitative measurement of bronchoscopic images. Eur Respir J 2001; 18: 817-826.

21 Carrol N, Elliot J, Morton A, James A. The structure of large and small airways in nonfatal and fatal asthma. Am Rev Respir Dis 1993; 147: 405-410.

22 Gillis HL, Lutchen KR. Airway remodeling in asthma amplifies heterogeneities in smooth muscle shortening causing hyperresponsiveness. J Appl Physiol 1999; 86: 2001-2012.

23 von Hayek H. The Human Lung. New York, Hafner, 1960; pp. 139-148.

24 Stephens NL, Jiang H. Basic Physiology of Airway Smooth Muscle. In: Busse WW, Holgate ST, eds. Asthma and Rhinitis. 1st Edn. UK, Blackwell Scientific Publications, 1995; pp. 1087-1089.

25 Mitchell HW, Gray PR. Uncoupling in the wall of the cartilaginous bronchus of the pig produced by smooth muscle contraction. Pulm Pharmacol 1996; 9: 29-34.

26 James AL, Hogg JC, Dunn LA, Paré PD. The use of the internal perimeter to compare airway size and to calculate smooth muscle shortening. Am Rev Respir Dis 1988; 138: 135-139.

27 Mitchell HW, Gray PR. Assessment of the dynamic relationship between external diameter and lumen flow in isolated bronchi. Respir Physiol 1999; 116: 67-76.

28 Ding DJ, Martin JG, Macklem PT. Effects of lung volume on maximal methacholine-induced bronchoconstriction in normal humans. J Appl Physiol 1987; 62: 1324-1330.
29 Brown RH, Mitzner W. Airway closure with high PEEP in vivo. J Appl Physiol 2000; 89: 956-960.

30 Mitchell HW, Cvetkovski R, Sparrow MP, Gray PR, McFawn PK. Concurrent measurement of smooth muscle shortening, lumen narrowing and flow to acetylcholine in large and small porcine bronchi. Eur Respir J 1998; 12: 1053-1061.

31 Okazawa M, Muller N, McNamara AE, Child S, Verburgt L, Paré PD. Human airway narrowing measured using high resolution computed tomography. Am J Respir Crit Care Med 1996; 154: 1557-1562.

32 Martin HB, Proctor DF. Pressure-volume measurements on dog bronchi. J Appl Physiol 1958; 13: 337-343.

33 Turner DJ, Noble PB, Lucas MP, Mitchell HW. Decreased airway narrowing and smooth muscle contraction in hyperresponsive pigs. J Appl Physiol 2002; 93: 1296-1300.

34 Noble PB, McFawn PK, Mitchell HW. Intraluminal pressure oscillation enhances subsequent airway contraction in isolated bronchial segments. J Appl Physiol 2004; 96: 1161-1165.

35 Khangure SR, Noble PB, Sharma A, Chia PY, McFawn PK, Mitchell HW. Cyclical elongation regulates contractile responses of isolated airways. J Appl Physiol 2004; 97: 913-919.

36 Hyatt RE, Offord KP, Lai-Fook SJ. Effect of length changes on bronchial diameters. J Appl Physiol 1981; 50: 1168-1172.

37 Hughes JMB, Hoppin FG Jr, Mead J. Effect of lung inflation on bronchial length and diameter in excised lungs. J Appl Physiol 1972; 32: 25-35.

38 Mead J, Takishima T, Leith D. Stress distribution in lungs: a model of pulmonary elasticity. J Appl Physiol 1970; 28: 596-608.

39 McFawn PK, Mitchell HW. Effect of transmural pressure on preloads and collapse of immature bronchi. Eur Respir J 1997; 10: 322-329.

40 Macklem PT. A theoretical analysis of the effect of airway smooth muscle load on airway narrowing. Am J Respir Crit Care Med 1996; 153: 83-89.

41 Lambert RK, Wilson TA. A model for the elastic properties of the lung and their effect on expiratory flow. J Appl Physiol 1973; 34: 34-48.

42 Mansell AL, Moalli RR, Calista CL, Pipkin AC. Elastic moduli of lungs during postnatal development in the piglet. J Appl Physiol 1989; 67: 1422-1427. 